\title{
Thérapie cellulaire et génique intracérébrale, trois échecs... prévisibles ?
}

En quelques mois, trois résultats négatifs d'essais cliniques, de thérapie cellulaire de la maladie de Parkinson d'une part $[1,2]$, de thérapie génique du glioblastome d'autre part [3], sont venus jeter le doute sur les possibilités de ces approches innovantes qui apparaissaient pourtant, il y a peu encore, si prometteuses. Le caractère spectaculaire de ces échecs a surtout été la conséquence de leur ampleur car, dans les trois cas considérés, les essais n'étaient plus présentés comme des « études pilotes «mais comme des essais à large échelle, contrôlés, de phase IIb voire III. Avant de juger les techniques de thérapie cellulaire et génique sur la base de ces résultats, pourtant, il faut s'intéresser à la méthodologie de chacun de ces essais pour en apprécier la validité. Dans les trois cas, en effet, on y trouve ce que l'on peut considérer comme des défauts majeurs, sur la base des connaissances expérimentales qui existaient, même, avant le début de l'étude.

\section{Règle $n^{\circ} 1$ : ne pas tuer les cellules lors de l'implantation}

La première étude publiée, chronologiquement, a été celle organisée par la compagnie Novartis sur la thérapie génique du glioblastome par implantation de cellules productrices de rétrovirus recombinants pour le gène thymidine kinase du virus $\mathrm{H}$ erpès simplex 1 (HSV-tk). Le glioblastome (qui touche chaque année près de 1500 personnes en France) est une tumeur maligne intracérébrale dont le pronostic est inéluctablement fatal, avec une médiane de survie qui ne dépasse pas 12 mois. Outre son insensibilité à la chimiothérapie anti- mitotique, le glioblastome est particulièrement résistant à la résection chirurgicale et à la radiothérapie du fait de la dispersion rapide des cellules dans le parenchyme nerveux central à partir de la masse tumorale, qui en fait une tumeur «localement métastatique». Comme cela a été décrit récemment dans $M$ édecine/ Sciences, la thérapie génique fondée sur l'introduction du gène suicide HSV-tk dans les cellules prolifératives, associée à l'administration systémique d'un faux substrat nucléotidique apporté par le ganciclovir, a montré une efficacité anti-tumorale intéressante in vitro et chez des modèles animaux de gliome [4]. Les résultats d'une étude pilote réalisée par l'équipe de David Klatzmann à I'Hôpital de la Pitié Salpêtrière (Paris) étaient, certes, hétérogènes mais pas totalement négatifs [5]. L'étude GL1328 organisée par Novartis s'appuyait donc sur ces travaux préliminaires, mais possédait une toute autre échelle puisque 248 patients étaient inclus dans une étude multicentrique, contrôlée et randomisée, réalisée dans une quarantaine de centres neurochirurgicaux nord-américains et européens. Le protocole de traitement était fondé sur le couplage d'une résection chirurgicale de la masse tumorale et de l'injection, consécutive, de cellules murines PA317 productrices d'un vecteur rétroviral dérivé du virus de la leucémie murine de Moloney, contenant le gène HSV-tk sous le contrôle du LTR et un gène neo utilisé pour la sélection des cellules productrices en G418. L'observation classique sur laquelle s'appuyait ce protocole complexe est le fait que les récidives post-chirurgicales des glio- blastomes ont lieu, dans la vaste majorité des cas, dans les régions d'infiltration les plus proches de la masse tumorale. L'analyse anatomopathologique retrouve en effet dans ces régions « péri-tumorales » le plus grand nombre de cellules tumorales infiltrées (même si une certaine proportion d'entre elles peuvent être beaucoup plus éloignées, dans les grands faisceaux de fibres nerveuses, ou le long des vaisseaux sanguins en particulier). L'implantation des cellules productrices dans les berges de la tumeur (à $1 \mathrm{~cm}$ de profondeur) visait donc à détruire sélectivement dans ces zones les cellules prolifératives, que leur mélange avec les cellules du parenchyme nerveux (postmitotiques pour l'essentiel) rend inaccessibles à l'exérèse chirurgicale. Les résultats de l'étude ont été totalement négatifs puisque la médiane de survie sans progression a été de 180 jours dans le groupe traité, de 183 jours dans le groupe contrôle, et la médiane de survie totale, respectivement de 365 et de 354 jours. Pourtant, et les auteurs eux-mêmes insistent sur ce point tout au long de leur discussion, cela ne permet pas d'inférer un échec de la thérapie génique en question parce que... la technique d'implantation des cellules était manifestement mauvaise ! En effet, le protocole prévoyait l'injection en 50 endroits différents, dans la paroi de la tumeur, de $200 \mu l$ d'une suspension contenant $10^{5}$ cellules par $\mu \mathrm{l}$. Ces chiffres faramineux (20 millions de cellules par site, 1 milliard de cellules au total) ont de quoi surprendre mais, surtout, de quoi inquiéter quant à la viabilité des cellules productrices de virus réellement implantées et, si les cellules n'ont pas sur- 
vécu à l'implantation, à la production de rétrovirus... Les neurochirurgiens qui ont décidé de ce protocole, apparemment en l'absence de biologistes et de spécialistes de greffes de cellules, n'ont pas réalisé ce que signifiait pour des cellules le fait d'être projetées, sous la pression de la seringue, contre le «mur de béton» que représente pour un tel volume le parenchyme cérébral. Lors de greffes de suspensions équivalentes de neurones fœtaux, I'administration est réalisée au rythme maximum de $5.10^{5}$ cellules par minute pour éviter l'effet des forces de convection à l'intérieur de l'aiguille d'une part, le choc contre la paroi d'autre part. Le volume est alors également limité à quelques $\mu \mathrm{l}$ de suspension par site, ce qui évite les reflux massifs (de cellules déjà mortes ?) que les auteurs de I'étude GL1328 rapportent comme systématiques. On peut s'étonner d'une faute méthodologique apparemment aussi grossière, et surtout de l'absence de validation par l'expérimentation animale qu'elle implique, mais il faut sans doute en tirer une conclusion banale: quand on fait " n'importe quoi » « n'importe comment», on n'arrive à rien.

\section{Règle $n^{\circ} 2$ : respecter les conditions expérimentales qui ont fait leur preuve}

C'est très probablement, même si les auteurs ne font pas dans ce cas leur autocritique, la même conclusion que l'on peut tirer de deux études de greffes neuronales appliquées à des patients atteints de la maladie de Parkinson dont les résultats viennent d'être présentés par des groupes américains différents $[1,2]$. Le premier concernait des allogreffes de neurones fœtaux humains, comme il s'en pratique dans de nombreux centres depuis $1990[6,7]$. De nouveau, la première particularité de cet essai - qui a largement stimulé la médiatisation de ses pauvres résultats tenait à son échelle ( 40 patients) et à son caractère randomisé et contrôlé, avec même dans ce cas un protocole en double-insu qui imposait une chirurgie fantôme aux patients du
Iarité de cette étude, toutefois, a éveillé l'inquiétude des spécialistes des greffes neuronales dès son lancement en 1994: elle ne respectait pas certaines des règles de base de la greffe neuronale, définies à partir de centaines de travaux expérimentaux, et elle ne reproduisait pas les protocoles qui avaient donné des résultats positifs dans les études cliniques pilotes. Les équipes européennes rassemblées dans le réseau NECTAR (network of european CNS transplantation and restoration) en étaient tellement surprises, et choquées, qu'elles avaient alors publié dans la revue Science une lettre commune mettant en cause le protocole, et indiquant dès alors que les résultats obtenus ne pourraient être tenus pour représentatifs des effets de cette thérapeutique, telle qu'elle est pratiquée ailleurs [8]. Malheureusement, la prédiction de l'époque s'est révélée exacte. Au contraire de ce que les études pilotes avaient montré, l'étude randomisée n'a abouti qu'à des effets limités chez les patients de moins de 60 ans, à aucun effet bénéfique du tout chez les plus âgés. Des effets indésirables graves ont même été rapportés, quelques malades présentant secondairement des dyskinésies incontrôlables.

Le principal défaut méthodologique de cette étude (mais il y en avait d'autres) était I'introduction, après le prélèvement des tissus chez les fœtus, d'une phase de culture de plusieurs semaines ( 3 à 4 ) préalables à l'implantation. Cette phase de culture tissulaire (en explants) contrevient directement à l'une des règles d'or de la greffe neuronale: I'absolue nécessité de respecter une fenêtre temporelle très précise pour l'âge du donneur. De très nombreuses études expérimentales ont en effet démontré que la survie des cellules implantées chute dramatiquement lorsque I'âge optimal - apprécié précisément chez I'homme entre 6 et, au grand maximum, 9 semaines après la conception pour les neurones dopaminergiques concernés ici - est dépassé. Les auteurs de l'étude américaine ont prélevé, comme le prescrivaient les conclusions des études expérimentales, les tissus chez des fœtus de 7 (ou 8) semaines après conception. Cependant, en les plaçant ensuite pour plusieurs semaines en culture, ils ont totalement négligé le fait que les cellules continuent alors à mûrir et qu'ils ont donc greffé, finalement, des cellules non pas de 7 ou 8 semaines, mais de 10 à 12 semaines après conception, c'està-dire parfaitement hors-limites. Les résultats qu'ils ont obtenus chez deux malades autopsiés, qui montraient 2000 à, au mieux, 20000 neurones dopaminergiques par trajet de greffe, confirment bien la validité des travaux expérimentaux - et a contrario la faute méthodologique commise puisque ces chiffres démontrent la perte de viabilité majeure du tissu. Ils sont en effet 5 à 70 (!) fois moins élevés que ceux obtenus par une autre équipe qui, utilisant la technique habituelle, en avait observé chez deux autres patients 90000 à 140000 par trajectoire [9]. Or, il existe une corrélation très nette entre le nombre de neurones implantés et l'étendue de la réinnervation dopaminergique du striatum, et une autre tout aussi nette entre la réinnervation et les effets cliniques [10]. Comme le disait le réseau NECTAR en 1994, et comme il le redit aujourd'hui dans une lettre adressée au journal qui a publié cette étude mal construite, tirer des conclusions générales est évidemment inapproprié.

\section{Règle $n^{\circ} 3$ : ne pas négliger les échecs expérimentaux}

Inapproprié, comme le serait sans doute une conclusion hâtive quant à l'impossibilité de réaliser des xénogreffes de neurones porcins chez des patients parkinsoniens, tirée de l'échec de l'étude menée par les sociétés Genzyme et Diacrin, dont les résultats totalement négatifs viennent d'être annoncés dans un communiqué de presse. Diacrin est une petite société de biotechnologie de Boston qui avait lancé, il y a 5 ans, une étude pilote de xénogreffes de neurones porcins chez des patients atteints soit de maladie de Parkinson, soit de maladie de Huntington. Le protocole de xénogreffe en lui-même n'est pas aberrant en ce qui concerne des greffes de neurones fœtaux, puisque 
de très nombreux travaux expérimentaux ont montré la possibilité de croiser diverses espèces, y compris le rat chez le primate [11], et I'homme chez le rat [12]. Ces études avaient souligné, toutefois, l'importance d'une immunosuppression lourde, les greffons ne survivant (et encore, seulement dans une proportion bien inférieure à des allogreffes) que si 10 à $15 \mathrm{mg} / \mathrm{kg}$ de ciclosporine A étaient administrés quotidiennement aux animaux, ce qui n'allait évidemment pas sans effet secondaire. Or, Diacrin avait, dans ses études pilotes, réduit l'immunosuppression en n'utilisant que des doses bien plus faibles de ciclosporine A ou même, chez d'autres malades, qu'un traitement par « masquage antigénique» dont I'intérêt n'avait pas été évident expérimentalement, mais I'était sans doute pour la société, qui en détenait les droits. Les études pilotes s'étant révélées globalement négatives [13]... un essai de phase IIb a été immédiatement lancé, en liaison avec la compagnie Genzyme. Cet essai, mené chez 20 patients dans des conditions d'immunosuppression pas mieux établies expérimentalement de fait, des chercheurs associés à ces essais disent maintenant que les études réalisées pour évaluer ces conditions d'immunosuppression chez le singe ont abouti systématiquement à un rejet de greffe ! - n'a pas donné mieux que les essais pilotes, et Genzyme a annoncé que les 20 millions de dollars prévus pour lancer une phase III en juin prochain avaient été réorientés vers d'autres circuits. II est vrai qu'on comprend le manque d'enthousiasme des financeurs lorsqu'on apprend que la plupart des études réalisées par tomographie en émission de positons à l'aide de ${ }^{18} \mathrm{~F}$-fluorodopa, destinées à évaluer la réinnervation dopaminergique dans le cerveau des patients, se sont elles aussi soldées par un résultat négatif, démontrant l'absence de survie significative des cellules greffées. Cela clôt, on peut l'espérer, la fuite en avant de Diacrin dans des essais de xénogreffes conçus sans études précliniques préalables suffisantes. En revanche, en tirer la conclusion que les xénogreffes de neurones porcins ne peuvent pas devenir un outil thé- rapeutique pour lutter contre les effets de la maladie de Parkinson serait à l'évidence abusif.

\section{Conclusions}

En conclusion de ce pauvre tour d'horizon d'erreurs méthodologiques grossières, on ne peut malheureusement que rappeler qu'il ne s'agissait pas d'expérimentations réalisées chez des rats Sprague-Dawley, mais chez des êtres humains souffrant de maladies très graves. Les (dizaines de) millions de dollars engloutis dans ces essais cliniques qui n'auraient pas dû être conduits, du moins pas de cette façon, auraient certainement été plus utiles dans une recherche expérimentale bien construite, bien menée et complète.

1. Freed CR, Greene PE, Breeze RE, et al. Trans plantation of embryonic dopamine neurons for severe Parkinson's disease. N Engl J Med 2001; $344: 710-9$.

2. http:/ / www.genzyme.com/ ir/ genz/ press/ 20010316.html

3. Rainov NG. A phase III clinical evaluation of Herpes simplex virus type 1 thymidine kinase and ganciclovir gene therapy as an adjuvant to surgical resection and radiation in adults with previously untreated glioblastoma multiforme. H uman Gene Ther 2000; 11: 2389-401.

4. Boyer 0 , Klatzmann $D$, Thérapie génique anticancéreuse par gènes suicides: du gène à l'essai clinique. M ed Sci 1999; 15: 625-34.

5. Klatzmann D, Valery CA, Bensimon G, et al. A phase I/II of herpes simplex virus type I thymidine kinase «suicide» gene therapy for recurrent glioblastoma. H uman Gene Ther 1998 : 9: 2595-604. 6 . Lindvall $\mathrm{O}$, Brundin $\mathrm{P}$, Widner $\mathrm{H}$, et al. Grafts of fetal dopamine neurons survive and improve motor function in Parkinson's disease. Science 1990; $247:$ 574-7.

7. Peschanski M, Defer G, Nguyen JP, et al. Bilateral motor improvement and alteration of $L$-dopa effect in two patients with Parkinson's disease following intrastriatal transplantation of fetal ventral mesencephalon. Brain 1994; 117: 487-99.

8. Widner $\mathrm{H}, \mathrm{NECTAR}$. NIH neural transplantation funding. Science $1994 ; 263: 737$.

9. Kordower JH, Freeman TB, Snow BJ, et al. Neuropathological evidence of graft survival and striatal reinnervation after the transplantation of fetal mesencephalic tissue in a patient with Parkinson's disease. N Engl J M ed 1995; 332: 1118-24.

10. Rémy $P$, Samson $Y, H$ antraye $P$, et al. Neural grafting in five parkinsonian patients: correlations between PET and clinical evolution. Ann Neurol 1995; 38: 580-8.
11. Hantraye P, Riche D, Maziere M, Isacson 0 . Intrastriatal transplantation of cross-species fetal striatal cells reduces abnormal movements in a primate model of $\mathrm{H}$ untington disease. Proc Natl Acad Sci USA 1992; 89: 4187-91.

12. Geny C, Naimi-Sadaoui S, Jeny R, Belkadi AM, Juliano SL, Peschanski M. Long-term delayed vascularization of human neural transplants to the rat brain. J Neurosci 1994 ; 14 : 7553-62.

13. Fink JS, Schumacher JM, Ellias SL, et al. Porcine xenografts in Parkinson's disease and Huntington's disease patients: preliminary results. Cell Transplant 2000; 9 : 273-8.

\section{Marc Peschanski}

Inserm U. 421, Faculté de médecine, 8, rue du Général-Sarrail, 94010 Créteil Cedex, France. 